\title{
Actividad antioxidante y antiproliferativa de seis plantas medicinales del noroeste de México
}

\author{
Antioxidant and antiproliferative activity of six medicinal plants from northwestern Mexico
}

\author{
Max Vidal Gutiérrez', Heriberto Torres Moreno², Carlos Arturo Velázquez Contreras', Luisa Alondra Rascón \\ Valenzuela ${ }^{1}$, Ramón Enrique Robles Zepeda ${ }^{1 *}$ \\ Universidad de Sonora, División de Ciencia Biológicas y de la Salud, Departamento de Ciencias Químico Biológicas, \\ Campus Hermosillo Sonora, México. \\ 2 Universidad de Sonora, División de Ciencias e Ingenierías, Departamento de Ciencias Químico Biológicas y Agropecuarias, \\ Unidad Regional Norte, Caborca Sonora, México.
}

\section{ABSTRACT}

Reactive oxygen species, which induce oxidative stress in the body, are one of the risk factors for some cancer types. In Mexico, the types of cancer with the highest mortality are prostate, breast, cervical, lung, and liver. Natural products represent the oldest form of medicinal remedies; for cancer, this source is no exception. In this investigation, we studied the antiproliferative activities, by MTT, and the antioxidant activity, by ABTS and DPPH, in six northwestern Mexico medicinal plants. The Jacquinia macrocarpa ssp. pungens fruit shells showed the best antiproliferative activity with an $\mathrm{IC}_{50}$ of $9.2 \mu \mathrm{g} / \mathrm{mL}$ in A549, followed by Bursera microphylla with $\mathrm{IC}_{50} 13.8 \mu \mathrm{g} / \mathrm{mL}$ in HeLa. Bursera laxiflora showed the best antioxidant activity of the six plants against ABTS and DPPH radicals, and also presented antiproliferative activity in HeLa. The bark of B. laxiflora contains compounds with antioxidant activities, which are probably also the same that induce antiproliferative activity in cancer cell lines. On the other hand, J. macrocarpa showed no antioxidant activity. However, the three species of plants represent a potential source of molecules with antiproliferative activity.

Keywords: Antiproliferative activity, Antioxidant activity, Bursera, Jacquinia, Acacia.

\section{RESUMEN}

Las especies reactivas de oxígeno, que inducen estrés oxidativo en el organismo, son uno de los factores de riesgo para padecer de algún tipo de cáncer. En México los tipos de cáncer con mayor mortalidad son el de próstata, mama, cervicouterino, pulmón e hígado. Los productos naturales representan la forma más antigua de remedios medicinales, para el cáncer esta fuente no es una excepción. En esta investigación, se estudió la actividad antioxidante por ABTS y DPPH, y la actividad antiproliferativa de seis plantas medicinales del noroeste de México. Las cáscaras del fruto de Jacquinia macrocarpa ssp pungens mostraron la mejor actividad antiproliferativa del estudio con una $\mathrm{IC}_{50}$ de $9.2 \mu \mathrm{g} /$ $\mathrm{mL}$ en A549, seguido por Bursera microphylla con IC ${ }_{50}$ de 13.8 $\mu \mathrm{g} / \mathrm{mL}$ en HeLa. Bursera laxiflora obtuvo la mejor actividad antioxidante de las seis plantas frente a los radicales ABTS y $\mathrm{DPPH}$, así como también presentó actividad antiproliferativa

Volumen XXII, Número 3 en HeLa. La corteza de B. laxiflora contiene compuestos con actividad antioxidante, que probablemente, también sean los mismos que inducen la actividad antiproliferativa en células cancerosas. Por otro lado, J. macrocarpa y B. microphylla no presentan actividad antioxidante, sin embargo, las tres especies vegetales representan una fuente potencial de moléculas con actividad antiproliferativa.

Palabras clave: Actividad antioxidante, Actividad antiproliferativa, Bursera, Jacquinia, Acacia.

\section{INTRODUCCIÓN}

El estrés oxidativo es uno de los factores de riesgo para padecer de cáncer, y sucede cuando los radicales libres en el organismo se encuentran en desequilibrio. Existen diferentes tipos de radicales libres, sin embargo, todos son capaces de dañar desde proteínas, hasta el propio ADN, pudiendo generar mutaciones que den origen a un cáncer (Moloney y Cotter, 2018). Según las estadísticas, en el año 2018, a nivel mundial se registraron 9.2 millones de defunciones a causa del cáncer. En México las cifras también son grandes, en 2015 se registraron 85201 muertes a causa de tumores malignos, donde se destacan el cáncer de próstata, mama, cervicouterino, pulmón e hígado como los principales responsables de dichos fallecimientos (Aldaco-Sarvide et al., 2019; Bray et al., 2018). El interés por encontrar medicamentos más eficientes contra el cáncer, se ha enfocado en el estudio de plantas que puedan contener moléculas o grupos de moléculas con potencial anticancerígeno. Estudios realizados sobre productos derivados de plantas del género Acacia, Bursera y Jacquinia, endémicas en México, indican que poseen actividad biológica contra diversas líneas celulares (LC) cancerosas. Sin embargo, también existen algunos estudios donde se reporta actividad antioxidante en estas especies (Afsar et al., 2018; Fon-Fay et al., 2019; Gigliarelli et al., 2018; Sadiq et al., 2017; Valenzuela-Cota et al., 2019). Es por ello que en esta investigación se seleccionó a Acacia cochliacantha, Acacia constricta, Bursera hindsiana, Bursera laxiflora, Bursera microphylla y Jacquinia macrocarpa ssp pungens, pertenecientes a la etnofarmacopea del noroeste de México, para determinar su potencial actividad antioxidante y antiproliferativo (Yetman y Van Devender, 2002).

*Autores para correspondencia: Ramón Enrique Robles Zepeda Correo electrónico: robles.zepeda@unison.mx

Recibido: 01 de diciembre de 2019 Aceptado: 22 de febrero de 2020 


\section{MATERIALES Y MÉTODOS \\ Equipos y reactivos}

Los radicales 2,2-diphenyl-1-picryhydrazyl (DPPH) y 2,2'-azino-bis(3-ethylbenzothiazoline-6-sulfonic acid) (ABTS) pertenecen a la marca Sigma-Aldrich, al igual que Dulbecco's Modified Eagle's Medium (DMEM), el 3-(4,5-dimethylthiazol-2-yl)-2,5-diphenyltetrazolium bromide (MTT), Trolox de Sigma-Aldrich y el Dimethyl sulfoxide (DMSO). Los equipos utilizados fueron los siguientes; incubadora de células eucariotas con control de temperatura a $37^{\circ} \mathrm{C}$ y mantenimiento de atmosfera al $5 \%$ de $\mathrm{CO}_{2}$ de la marca Thermo Scientific, campana de flujo laminar equipada con filtros HEPA y lector de placas BMG LABTECH's POLARstar Omega.

\section{Líneas celulares y cultivo celular}

Las LC L929 (tejido conectivo subcutáneo murino normal) y HeLa (carcinoma humano de cérvix) fueron obtenidas por parte de la American Type Culture Collection (ATCC), A594 (carcinoma alveolar humano), M12Ak.C3.F6 (linfoma de células B murinas) y RAW 264.7 (leucemia de macrófagos transformadas por el virus de Albenson) fueron proporcionadas por el Dr. Emil A. Unanue del Departamento de Patología e Inmunología de la Universidad de Washington. Todas las células fueron cultivadas en el medio de cultivo DMEM suplementado al $5 \%$ con suero fetal bovino (D5F), en una atmósfera al $5 \%$ de $\mathrm{CO}_{2}$ a $37^{\circ} \mathrm{C}$.

\section{Obtención de resinas y extractos metanólicos}

Las resinas de $B$. hindsiana y $B$. microphylla fueron colectadas directamente de los troncos de plantas ubicadas en la región de Bahía de Kino, en Hermosillo Sonora, México. Los extractos metanólicos fueron obtenidos a partir de las partes aéreas de $A$. cochliacantha, de A. constricta, y de J. macrocarpa ssp pungens, la corteza de B. laxiflora y las cáscaras del fruto de J. macrocarpa ssp punges. El material vegetal fue secado a temperatura ambiente para posteriormente ser molido en un molino tipo Wiley, y reposar en metanol $(\mathrm{MeOH})$ a una relación 1:10 p/v de material vegetal durante 10 días, con periodos regulares de agitación. La solución metanólica obtenida se sometió a filtración para finalmente secarla a presión reducida a $40^{\circ} \mathrm{C}$ por rotaevaporación. El extracto crudo resultante fue secado a temperatura ambiente y almacenado en recipientes color ámbar $\mathrm{a}-4{ }^{\circ} \mathrm{C}$ hasta su uso posterior.

\section{Capacidad reductora del radical ABTS}

Se prepararon soluciones de las muestras a concentraciones seriadas de $200 \mu \mathrm{g} / \mathrm{mL}$ a $12.5 \mu \mathrm{g} / \mathrm{mL}$, utilizando etanol (EtOH) como disolvente. Fueron disueltos $19.3 \mathrm{mg}$ de ABTS en $5 \mathrm{~mL}$ de $\mathrm{H}_{2} \mathrm{O}$, a esta disolución se le agregaron 88 $\mu \mathrm{L}$ de $\mathrm{K}_{2} \mathrm{~S}_{2} \mathrm{O}_{8} 140 \mu \mathrm{M}$ y se dejó reposar por $16 \mathrm{~h}$ en oscuridad a temperatura ambiente. Posteriormente se preparó una solución con EtOH a 0.7 de absorbancia con una longitud de onda de $730 \mathrm{~nm}$, utilizando EtOH como blanco. Se colocaron $295 \mu \mathrm{L}$ de la solución en los pozos de una placa de 96, al cual se le agregaron $5 \mu \mathrm{L}$ de las soluciones de extractos y resinas diluidas en $\mathrm{EtOH}$, se dejó reposar por 5 min a temperatura ambiente y finalmente se realizaron las lecturas en un lector de placas, utilizando una longitud de onda de $730 \mathrm{~nm}$. Los resultados son expresados en microgramos equivalentes a trolox $(\mu g E T / \mathrm{mL})$ utilizando el mismo procedimiento para construir una recta con el antioxidante trolox a concentraciones de 10 a $200 \mu \mathrm{g} / \mathrm{mL}$ (Sridhar y Charles, 2019).

\section{Capacidad reductora del radical DPPH}

Se prepararon soluciones de las muestras a concentraciones seriadas de $200 \mu \mathrm{g} / \mathrm{mL}$ a $12.5 \mu \mathrm{g} / \mathrm{mL}$, utilizando $\mathrm{MeOH}$ como disolvente. El reactivo DPPH fue disuelto en $\mathrm{MeOH}$ y ajustado a una absorbancia de 0.7 a $515 \mathrm{~nm}$. En una placa de 96 pozos se colocaron $280 \mu \mathrm{L}$ de la solución $\mathrm{DPPH} / \mathrm{MeOH}$ y se le adicionaron $20 \mu \mathrm{L}$ de las soluciones de las muestras (volumen final $300 \mu \mathrm{L}$ ). Posteriormente, esta solución se dejó reposar por 30 min en la oscuridad a temperatura ambiente. Por último, la placa fue leída a una longitud de onda de 515 $\mathrm{nm}$ en un lector de placas, utilizando $\mathrm{MeOH}$ como blanco. Se realizó el mismo procedimiento para la construcción de una curva de actividad antioxidante del trolox a concentraciones de $100 \mu \mathrm{g} / \mathrm{mL}$ a $5 \mu \mathrm{g} / \mathrm{mL}$, y se expresaron los resultados en $\mu \mathrm{gET} / \mathrm{mL}$ (Sridhar y Charles, 2019).

\section{Medición de la Viabilidad Celular Mediante el Ensayo MTT}

El ensayo del MTT se basa en la reducción metabólica realizada por la mitocondria sobre este compuesto por la enzima succinato-deshidrogenasa, formando un compuesto coloreado llamado formazán. De esta manera se determina la funcionalidad de las mitocondrias de las células tratadas. La cantidad de células vivas es proporcional a la cantidad de formazán obtenido. Para evaluar la actividad antiproliferativa de las muestras, los extractos y resinas fueron disueltos en DMSO a $40 \mathrm{mg} / \mathrm{mL}$, y posteriormente en D5F realizando soluciones seriadas de $400 \mu \mathrm{g} / \mathrm{mL}$ a $6.25 \mu \mathrm{g} / \mathrm{mL}$. Se preparó una suspensión celular de $200,000 \mathrm{cel} / \mathrm{mL}$ que se distribuyó en una placa de 96 pozos colocando $50 \mu \mathrm{L}$ de la suspensión en cada pozo. Se incubó la placa a $37^{\circ} \mathrm{C}$ y atmósfera de $5 \%$ de $\mathrm{CO}_{2}$ por un tiempo de $24 \mathrm{~h}$. Posteriormente, se agregó a cada pozo $50 \mu \mathrm{L}$ de las diluciones de las muestras y se dejó incubar en condiciones de cultivo por $48 \mathrm{~h}$, con observaciones periódicas al microscopio en lapsos de 24 h a 48 h. Después de haber hecho las observaciones pertinentes en un lapso de 48 h, se realizó un lavado a la placa con una solución de soporte de fosfatos 1 X (PBS 1X) (excepto a las células M12Ak. C3.F6) y se añadió nuevamente $100 \mu \mathrm{L}$ de D5F. Finalmente, se agregaron $10 \mu \mathrm{L}$ de una solución de MTT de $5 \mathrm{mg} / \mathrm{mL}$ en PBS 1X y se dejó reposar por $4 \mathrm{~h}$ en condiciones de cultivo. Una vez concluido el tiempo de reposo, los cristales de formazán formados se disolvieron en $100 \mu \mathrm{L}$ de isopropanol acidificado y las lecturas se realizaron en un lector de placas a una longitud de onda dual de $570 \mathrm{~nm}$ y $630 \mathrm{~nm}$. Se utilizó como control concentraciones de DMSO de $0.01-0.25 \%$ en las LC, las cuales no mostraron ningún tipo de daño celular (Torres-Moreno et al., 2015). 


\section{Índice de Selectividad}

El índice de selectividad de los extractos y resinas, se refiere a la relación de la susceptibilidad a la inhibición del crecimiento entre la $\mathrm{LC}$ normal y la $\mathrm{LC}$ cancerosa: $\mathrm{IC} \mathrm{C}_{50} \mathrm{LC}$ normal / IC ${ }_{50}$ LC cancerosa. En la cual, $>1$; indica que el tratamiento es selectivo hacia la LC cancerosa, y $<1$ cuando el tratamiento tiene mayor selectividad por la LC no cancerosa (Torres-Moreno, 2017)

\section{RESULTADOS Y DISCUSIONES Actividad antioxidante}

El ABTS es un sustrato de la peroxidasa, que al oxidarse en presencia de $\mathrm{H}_{2} \mathrm{O}_{2}$ se produce el catión radical ABTS.+ En solución, este radical presenta un color verde intenso y puede ser monitoreado por espectrofotometría en un intervalo de longitud de onda de $600-750 \mathrm{~nm}$. Al reaccionar el catión ABTS.+ con un antioxidante, éste se neutraliza a través de la captación de un electrón por parte del agente antioxidante. La neutralización del radical ABTS.+ en solución, se percibe por la decoloración gradual del verde intenso (Karadag et al., 2009). Por otro lado, el radical DPPH tiene la característica de presentar un electrón desapareado en su estructura, que en solución presenta un color púrpura. La absorbancia máxima del DPPH en solución se presenta a una longitud de onda 515-517 nm en el espectrofotómetro. Estas características cambian cuando el DPPH acepta un protón, estabilizando la estructura del radical a DPPH-H, el cual presenta un color amarillo en solución (Tirzitis y Bartosz, 2010).

Los extractos metanólicos de A. cochliacantha y B. laxiflora tienen un comportamiento de actividad antioxidante dosis dependiente, tanto para el método ABTS (tabla 1) como para el DPPH (tabla 2). En el resto de las muestras, la actividad antioxidante se mantiene estable sin diferencias estadísticamente significativas entre las concentraciones evaluadas. Los resultados obtenidos en ambas especies de Acacia, a pesar de pertenecer al mismo género, muestran que la capacidad estabilizadora de radicales libres no es igual, esto debido a que $A$. conchliacantha tiene mayor actividad antioxidante que $A$. constricta. Otra diferencia se observa al comparar los resultados obtenidos de $A$. cochliacantha y $A$. constricta, con los publicados por Agrawal et al. (2010) para Acacia nilotica. En este artículo se reporta una fuerte actividad antioxidante sobre el radical DPPH, donde a una concentración de $10 \mu \mathrm{g} /$ $\mathrm{mL}$ neutraliza más del $90 \%$ del radical. Para que A. cochliacantha pueda estabilizar la mitad de lo que $A$. nilotica inhibe con $10 \mu \mathrm{g} / \mathrm{mL}$, es necesaria una concentración veinte veces mayor, es decir, A. cochliacantha tiene la capacidad de estabilizar el $45 \%$ del radical DPPH solo a $200 \mu \mathrm{g} / \mathrm{mL}$ (tabla 3 ). Esto sugiere que la cantidad de antioxidantes que contiene el género Acacia, no es homogénea entre sus especies (Agrawal et al., 2010).

B. laxiflora presentó la mayor actividad antioxidante de las seis plantas evaluadas, en cambio, la capacidad estabilizadora de radicales libres de $B$. hindsiana y $B$. microphylla se mantuvo estable en todas las concentraciones estudiadas para ABTS, y sin capacidad estabilizadora del radical DPPH.
Tabla 1. Actividad antioxidante en $\mu \mathrm{gET} / \mathrm{mL}^{*}$ de extractos metanólicos y resinas por el método ABTS.

Table 1. Antioxidant activity in $\mu \mathrm{gET} / \mathrm{mL}^{*}$ of methanolic extracts and resins by the ABTS method.

\begin{tabular}{|c|c|c|c|c|c|}
\hline \multirow[b]{2}{*}{ Planta } & \multicolumn{5}{|c|}{ Concentración en $\mu \mathrm{g} / \mathrm{mL}^{* *}$} \\
\hline & 200 & 100 & 50 & 25 & 12.5 \\
\hline $\begin{array}{l}\text { A. cochlia } \\
\text { cantha }\end{array}$ & $\begin{array}{l}63.7 \pm \\
11.4^{\mathrm{A}, \mathrm{b}}\end{array}$ & $\begin{array}{c}41.7 \pm \\
12^{\mathrm{B}, \mathrm{b}}\end{array}$ & $\begin{array}{c}24 \pm \\
3.2^{\mathrm{BC}, \mathrm{b}}\end{array}$ & $\begin{array}{c}22 \pm \\
3.1^{\mathrm{BC}, \mathrm{a}}\end{array}$ & $\begin{array}{c}18.8 \pm \\
2.8^{\mathrm{C}, a}\end{array}$ \\
\hline A. constricta & $\begin{array}{l}26.8 \pm \\
3.0^{\mathrm{A}, \mathrm{c}}\end{array}$ & $\begin{array}{r}22.7 \pm \\
4.5^{A, b}\end{array}$ & $\begin{array}{l}21.3 \pm \\
2.3^{\mathrm{A}, \mathrm{b}}\end{array}$ & $\begin{array}{l}19.4 \pm \\
3.3^{\mathrm{A}, \mathrm{a}}\end{array}$ & $\begin{array}{l}17.9 \pm \\
3.9^{\mathrm{A}, \mathrm{a}}\end{array}$ \\
\hline B. hindsiana ${ }^{\S}$ & $\begin{array}{l}22.9 \pm \\
11.6^{A, c}\end{array}$ & $\begin{array}{l}22.0 \pm \\
11.4^{\mathrm{A}, \mathrm{b}}\end{array}$ & $\begin{array}{l}17.1 \pm \\
4.5^{\mathrm{A}, \mathrm{b}}\end{array}$ & $\begin{array}{c}17.7 \pm \\
5.3^{\mathrm{A}, \mathrm{a}}\end{array}$ & $\begin{array}{l}17.6 \pm \\
4.0^{\mathrm{A}, \mathrm{a}}\end{array}$ \\
\hline B. laxiflora & $\begin{array}{c}105.8 \pm \\
5.3^{\mathrm{A}, \mathrm{a}}\end{array}$ & $\begin{array}{c}63.9 \pm \\
2.3^{\mathrm{B}, \mathrm{a}}\end{array}$ & $\begin{array}{l}41.2 \pm \\
2.8^{\mathrm{C}, \mathrm{a}}\end{array}$ & $\begin{array}{c}30.6 \pm \\
4.1^{\mathrm{D}, \mathrm{a}}\end{array}$ & $\begin{array}{c}28.7 \pm \\
1.1^{\mathrm{D}, \mathrm{a}}\end{array}$ \\
\hline B. microphylla ${ }^{\S}$ & $\begin{array}{c}20.5 \pm \\
4.9^{A, c}\end{array}$ & $\begin{array}{c}20.1 \pm \\
4.2^{A, b}\end{array}$ & $\begin{array}{c}18.7 \pm \\
4.0^{\mathrm{A}, \mathrm{b}}\end{array}$ & $\begin{array}{c}19.0 \pm \\
6.1^{\mathrm{A}, \mathrm{a}}\end{array}$ & $\begin{array}{l}18.4 \pm \\
4.4^{\mathrm{A}, \mathrm{a}}\end{array}$ \\
\hline $\begin{array}{l}\text { J. macro } \\
\text { carpa ssp. } \\
\text { pungens }{ }^{\text {CF }}\end{array}$ & $\begin{array}{c}24.7 \pm \\
6.1^{A, c}\end{array}$ & $\begin{array}{l}21.3 \pm \\
8.0^{\mathrm{A}, \mathrm{b}}\end{array}$ & $\begin{array}{l}18.1 \pm \\
9.1^{\mathrm{A}, \mathrm{b}}\end{array}$ & $\begin{array}{l}16.9 \pm \\
7.5^{\mathrm{A}, \mathrm{a}}\end{array}$ & $\begin{array}{l}15.6 \pm \\
8.5^{A, a}\end{array}$ \\
\hline $\begin{array}{l}\text { J. macro } \\
\text { carpa ssp. } \text { pungens } \\
\text { pA }\end{array}$ & $\begin{array}{c}26.2 \pm \\
5.4^{A, c}\end{array}$ & $\begin{array}{l}21.7 \pm \\
7.6^{A, b}\end{array}$ & $\begin{array}{l}18.2 \pm \\
6.8^{\mathrm{A}, \mathrm{b}}\end{array}$ & $\begin{array}{l}17.6 \pm \\
7.9^{\mathrm{A}, \mathrm{a}}\end{array}$ & $\pm 7.7^{\mathrm{A}, \mathrm{a}}$ \\
\hline
\end{tabular}

* Microgramos equivalentes a Trolox por mililitro de muestra ( $\mu \mathrm{gET} /$ $\mathrm{mL}$ ) / Trolox Equivalent micrograms by mililiter of sample; $\mathrm{R}^{2}=0.9942$, $\mathrm{y}=0.4166 \mathrm{x}+2.9198$.

* Los valores obtenidos representan tres experimentos independientes \pm su desviación estándar / The values obtained represent three independent experiments \pm standard deviation.

\& Resinas / Resins.

CF Cáscaras de fruto / Fruit peles.

PA Partes aéreas / Aerial parts.

A-D Diferencias estadísticamente significativas entre las concentraciones de cada especie vegetal (horizontal) / Statistically significant differences between the concentrations of each plant species (horizontal). ANOVA one-way; post-hoc Tukey $(\mathrm{p}<0.05)$.

a-c Diferencias estadísticamente significativas entre las especies vegetales de cada concentración (vertical)/ Statistically significant differences between plant species to be found in each concentration (vertical). ANOVA one-way; post-hoc Tukey $(\mathrm{p}<0.05)$.

Un estudio de actividad antioxidante realizado sobre el extracto metanólico de corteza de Bursera simaruba indica una pobre capacidad de neutralizar a los radicales ABTS y DPPH, pues bien, la concentración en la que neutraliza el $50 \%$ del radical es de $0.525 \mathrm{mg} / \mathrm{mL}$ y $0.506 \mathrm{mg} / \mathrm{mL}$ para ABTS y DPPH respectivamente (Bah et al., 2014). En cambio, el extracto metanólico de la corteza de B. laxiflora, a $200 \mu \mathrm{g} /$ $\mathrm{mL}$, neutraliza el $46.9 \%$ del radical ABTS, y con $100 \mu \mathrm{g} / \mathrm{mL}$, el $50.2 \%$ del radical DPPH (tabla 3 ). Esto puede significar que los compuestos antioxidantes de Bursera se mantienen en la corteza, y al momento de formarse las resinas los antioxidantes no son secretados, o bien, estos si se encuentran presentes en las resinas, pero ya en su forma oxidada.

\section{Actividad Antiproliferativa e Índice de Selectividad}

Ciertos antioxidantes, como lo es el grupo de los flavonoides, son conocidos por su capacidad estabilizadora de radicales libres. Sin embargo, algunas moléculas de este grupo tienen la capacidad de inducir mecanismos que activan la muerte celular en células cancerosas, e inhibir la proliferación 
Tabla 2. Actividad antioxidante en $\mu \mathrm{gET} / \mathrm{mL}^{*}$ de extractos metanólicos y resinas por el método DPPH.

Table 2. Antioxidant activity in $\mu \mathrm{gET} / \mathrm{mL}^{*}$ of methanolic extracts and resins by the DPPH method.

\begin{tabular}{|c|c|c|c|c|c|}
\hline \multirow[b]{2}{*}{ Planta } & \multicolumn{5}{|c|}{ Concentración en $\mu \mathrm{g} / \mathrm{mL}^{* *}$} \\
\hline & 200 & 100 & 50 & 25 & 12.5 \\
\hline A.cochliacantha & $\begin{array}{l}41.5 \pm \\
0.3^{\mathrm{A}, \mathrm{b}}\end{array}$ & $\begin{array}{l}20.4 \pm \\
1.6^{\mathrm{B}, \mathrm{b}}\end{array}$ & $\underset{c, b}{8.6 \pm 1.1}$ & $\underset{D, b}{2.0 \pm 1.3}$ & $0.0^{\mathrm{D}, \mathrm{b}}$ \\
\hline A.constricta & $\underset{A, d}{2.2 \pm 1.5}$ & $\begin{array}{c}0.2 \pm 2.4 \\
A, C\end{array}$ & $\begin{array}{c}0.7 \pm 2.5 \\
A, C\end{array}$ & $0.0^{\mathrm{A}, \mathrm{c}}$ & $0.0^{\mathrm{A}, \mathrm{b}}$ \\
\hline B. hindsiana ${ }^{\S}$ & $0.0^{\mathrm{A}, \mathrm{d}}$ & $0.0^{A, c}$ & $0.0^{A, C}$ & $0.0^{\mathrm{A}, \mathrm{C}}$ & $0.0^{\mathrm{A}, \mathrm{b}}$ \\
\hline B. laxiflora & $\begin{array}{l}95.0 \pm \\
0.7^{\mathrm{A}, \mathrm{a}}\end{array}$ & $\begin{array}{l}47.8 \pm \\
0.7^{B, a}\end{array}$ & $\begin{array}{l}23.6 \pm \\
0.8^{\mathrm{C} a \mathrm{a}}\end{array}$ & $\begin{array}{c}7.4 \pm 0.2 \\
D, a\end{array}$ & $\begin{array}{c}3.7 \pm 0.6 \\
E, a\end{array}$ \\
\hline B. microphylla $a^{\S}$ & $0.0^{\mathrm{A}, \mathrm{d}}$ & $0.0^{\text {A.c }}$ & $0.0^{A, C}$ & $0.0^{A, C}$ & $0.0^{\mathrm{A}, \mathrm{b}}$ \\
\hline $\begin{array}{l}\text { J. macrocarpa } \\
\text { ssp. pungens }{ }^{\mathrm{CF}}\end{array}$ & $\underset{A, C}{5.9 \pm 2.4}$ & $\begin{array}{l}0.04 \pm \\
2.2^{B, c}\end{array}$ & $0.0^{\mathrm{B}, \mathrm{C}}$ & $0.0^{B, C}$ & $0.0^{\mathrm{B}, \mathrm{b}}$ \\
\hline $\begin{array}{l}\text { Jacquinia } \\
\text { macrocarpa } \\
\text { ssp.pungens }{ }^{\mathrm{PA}}\end{array}$ & $0.0^{\mathrm{A}, \mathrm{d}}$ & $0.0^{A, C}$ & $0.0^{A, c}$ & $0.0^{A, C}$ & $0.0^{\mathrm{A}, \mathrm{b}}$ \\
\hline
\end{tabular}

* Microgramos equivalentes a Trolox por mililitro de muestra $(\mu \mathrm{gET} / \mathrm{mL}) /$ Trolox Equivalent micrograms by mililiter of sample; $R^{2}=0.9942, y=0.4166 x$ $+2.9198$

** Los valores obtenidos representan tres experimentos independientes $\pm \mathrm{su}$ desviación estándar / The values obtained represent three independent experiments \pm standard deviation

$\S$ Resinas / Resins.

CF Cáscaras de fruto / Fruit peels

PA Partes aéreas / Aerial parts.

A-E Diferencias estadísticamente significativas entre las concentraciones de cada especie vegetal (horizontal) / Statistically significant differences between the concentrations of each plant species (horizontal). ANOVA oneway; post-hoc Tukey $(p<0.05)$.

a-c Diferencias estadísticamente significativas entre las especies vegetales de cada concentración (vertical) / Statistically significant differences between plant species to be found in each concentration (vertical). ANOVA one-way; post-hoc Tukey $(p<0.05)$.

Tabla 3. Actividad antioxidante en porcentaje de inhibición de los radicales ABTS y DPPH de extractos y resinas.

Table 3. Antioxidant activity in percent inhibition of ABTS and DPPH radicals of extracts and resins.

\begin{tabular}{|c|c|c|c|c|c|c|c|c|c|c|}
\hline \multirow{3}{*}{ Planta / Radical } & \multicolumn{10}{|c|}{ Concentración $\mu \mathrm{g} / \mathrm{mL}$} \\
\hline & \multicolumn{2}{|c|}{200} & \multicolumn{2}{|c|}{100} & \multicolumn{2}{|c|}{50} & \multicolumn{2}{|c|}{25} & \multicolumn{2}{|c|}{12.5} \\
\hline & DPPH & ABTS & DPPH & ABTS & DPPH & ABTS & DPPH & ABTS & DPPH & ABTS \\
\hline $\begin{array}{l}\text { A. cochlia } \\
\text { cantha }\end{array}$ & 45.4 & 29.5 & 29.4 & 20.3 & 20.4 & 12.9 & 15.4 & 12.1 & 12.5 & 10.7 \\
\hline A. constricta & 15.5 & 14.1 & 14.1 & 12.4 & 14.5 & 11.8 & 12.4 & 11 & 11.6 & 10.3 \\
\hline B. hindsiana ${ }^{5}$ & 10.5 & 12.5 & 10.5 & 12.1 & 10.8 & 10.1 & 10.3 & 10.3 & 10.8 & 10.2 \\
\hline B. laxiflora & 85.9 & 46.9 & 50.2 & 29.6 & 31.8 & 20.1 & 19.5 & 15.7 & 16.7 & 14.9 \\
\hline B. microphylla ${ }^{\S}$ & 11.1 & 12.3 & 11.7 & 12.2 & 10.9 & 11.2 & 11.5 & 11.6 & 10.6 & 11.2 \\
\hline $\begin{array}{l}\text { J. macrocarpa } \\
\text { ssp. pungens } \\
\text { (cáscara fruto) }\end{array}$ & 17.1 & 13.2 & 13.9 & 11.8 & 12.9 & 10.4 & 11.8 & 9.8 & 11.6 & 9.4 \\
\hline $\begin{array}{l}\text { J. macrocarpa } \\
\text { ssp. pungens } \\
\text { (partes aéreas) }\end{array}$ & 12.5 & 13.85 & 11.7 & 11.9 & 11.5 & 10.5 & 11.5 & 10.2 & 11.6 & 9.84 \\
\hline
\end{tabular}

Los valores tabulados representan el porcentaje de inhibición del radical indicado con la concentración del extracto señalada / tabulated values represent the percentage of inhibition of the radical indicated with the concentration of the extract indicated $\S$ Resinas / Resins. celular. Estos polifenoles modulan vías de señalización paralizando la progresión y desarrollo del cáncer, así como activando señales de muerte celular que pueden inducir la apoptosis en las células malignas y pre-cancerosas (Abbas et al., 2017).

El extracto metanólico de las cáscaras de fruto de $J$. macrocarpa ssp.pungens exhibe la mejor actividad antiproliferativa en las cuatro LC cancerosas (tabla 4) comparado con las otras muestras evaluadas. Adicionalmente, la LC menos susceptible al extracto es $\mathrm{L} 929\left(\mathrm{IC}_{50} 47.8 \mu \mathrm{g} / \mathrm{mL}\right)$, lo que indica un comportamiento del tipo selectivo contra las LC cancerosas (tabla 5). Este comportamiento también lo presenta el extracto metanólico de las partes aéreas de la misma planta, pero con valores de $\mathrm{IC}_{50}$ más elevados. Estudios de actividad antiproliferativa realizadas sobre Bonellia albiflora (Jacquinia albiflora), reportan, que el extracto metanólico de hojas po- see una $I_{50}$ de $47.05 \mu \mathrm{g} / \mathrm{mL}$ en HeLa (Moo-Puc et al., 2013), lo que demuestra que su actividad en esta LC es mayor que el extracto de partes aéreas de $J$. macrocarpa ssp. pungens, pero con valores próximos a los observados para el extracto de las cáscaras del fruto. Es poco probable que los compuestos responsables por la actividad antiproliferativa en Jacquinia sean compuestos fenólicos, esto debido a que los extractos evaluados no poseen actividad antioxidante, además, solo se han reportado triterpenos glucosilados para especies de este género (García-Sosa et al., 2011; Vila-Luna et al., 2017), por lo que probablemente, este tipo de compuestos sean los responsables por la actividad antiproliferativa en las LC cancerosas (Sofi et al., 2018).

El género Bursera es estudiado actualmente por sus propiedades biológicas en sistemas in vitro. En el presente estudio, podemos observar actividades antiproliferativas im- 
Tabla 4. Actividad antiproliferativa de seis plantas medicinales.

Table 4. Antiproliferative activity of six medicinal plants.

\begin{tabular}{|c|c|c|c|c|c|}
\hline \multirow[t]{2}{*}{ Planta } & \multicolumn{5}{|c|}{ IC50 en $\mu \mathrm{g} / \mathrm{mL}^{*}$} \\
\hline & A549 & HeLa & M12Ak.C3.F6 & RAW264.7 & L929 \\
\hline A. cochliacantha & $N D^{* *}$ & $164.7 \pm 35^{e}$ & $113.8 \pm 14.5^{e}$ & $168.9 \pm 15.5^{d}$ & $120.4 \pm 9.1^{c}$ \\
\hline A. constricta & $N D^{* *}$ & $116.7 \pm 3.1^{d}$ & $110.6 \pm 13.3^{e}$ & $112.6 \pm 7.1^{c}$ & $115.2 \pm 2.2^{c}$ \\
\hline B. hindsiana ${ }^{5}$ & $91.0 \pm 5.6^{d}$ & $95.3 \pm 2.4^{c}$ & $60.4 \pm 4.7^{c}$ & $58.7 \pm 2.3^{b}$ & $96.8 \pm 1.3^{b}$ \\
\hline B. laxiflora & $198.2 \pm 7.4^{e}$ & $93.7 \pm 5.4^{c}$ & $98.0 \pm 3.2^{d}$ & $117.4 \pm 1.5^{c}$ & $N D^{* *}$ \\
\hline B. microphylla ${ }^{\S}$ & $53.8 \pm 1.4^{c}$ & $13.8 \pm 1.4^{\mathrm{a}}$ & $26.0 \pm 2.1^{\mathrm{a}}$ & $21.9 \pm 3.1^{\mathrm{a}}$ & $39.3 \pm 0.9^{a}$ \\
\hline $\begin{array}{l}\text { J. macrocarpa ssp. } \\
\text { pungens (cáscara fruto) }\end{array}$ & $9.2 \pm 2.2^{\mathrm{a}}$ & $40.8 \pm 2.3^{b}$ & $22.0 \pm 2.0^{\mathrm{a}}$ & $26.6 \pm 1.4^{a}$ & $47.8 \pm 2.0^{a}$ \\
\hline $\begin{array}{l}\text { J. macrocarpa ssp. } \\
\text { pungens (partes aéreas) }\end{array}$ & $45.4 \pm 0.6^{b}$ & $85.5 \pm 4.4^{c}$ & $45.0 \pm 2.0^{b}$ & $47.7 \pm 0.6^{b}$ & $87.2 \pm 4.0^{b}$ \\
\hline \multicolumn{6}{|c|}{$\begin{array}{l}\text { ND; IC50 no detectada a la máxima concentración evaluada }(200 \mu \mathrm{g} / \mathrm{mL}) / \text { IC5O Not detected the maximum } \\
\text { concentration evaluated }(200 \mu \mathrm{g} / \mathrm{mL}) \\
\text { Resinas / Resins. }\end{array}$} \\
\hline \multicolumn{6}{|c|}{$\begin{array}{l}\text { a-e Diferencias estadísticamente significativas entre las IC50 de las especies vegetales en la misma línea celular } \\
\text { (vertical) / Statistically significant differences between the IC50 of the plant species in the same cell line (vertical) } \\
\text { ANOVA one-way; post-hoc Tukey }(p<0.05) \text {. }\end{array}$} \\
\hline
\end{tabular}

Tabla 5. Índice de selectividad de extractos metanólicos y resinas en las líneas celulares cancerosas a .

Table 5. Selectivity index of methanolic extracts and resins in the cancer cell lines a.

\begin{tabular}{|c|c|c|c|c|}
\hline \multirow[t]{2}{*}{ Planta } & \multicolumn{4}{|c|}{ Líneas celulares cancerosas ${ }^{b, c}$} \\
\hline & A549 & HeLa & M12Ak.C3.F6 & RAW264.7 \\
\hline A. cochliacantha & $<1$ & 0.73 & 1.06 & 0.71 \\
\hline A. constricta & $<1$ & 0.99 & 1.04 & 1.02 \\
\hline B. hindsiana ${ }^{\S}$ & 1.06 & 1.01 & 1.6 & 1.65 \\
\hline B. laxiflora & $>1$ & $>1$ & $>1$ & $>1$ \\
\hline B. microphylla ${ }^{\S}$ & 0.73 & 2.85 & 1.51 & 1.79 \\
\hline J. macrocarpa ssp. pungens ${ }^{C F}$ & 5.2 & 1.17 & 2.17 & 1.8 \\
\hline J. macrocarpa ssp. pungens ${ }^{P A}$ & 1.92 & 1.02 & 1.94 & 1.83 \\
\hline
\end{tabular}

a Relación de actividad antiproliferativa de las células cancerosas respecto a la línea celular no cancerosa / Relationship of antiproliferative activity of cancer cells with respect to the non-cancerous cell line

b Valores $<1$; Selectividad sobre línea celular normal / Values $<1$; Selectivity on the normal cell line.

c Valores $>1$; Selectividad sobre línea celular cancerosa / Values $>1$; Selectivity on cancer cell line.

$\$$ Resinas/Resins.

CF Cáscaras de fruto / Fruit peels.

PA Partes aéreas / Aerial parts.

portantes en las tres especies de Bursera, siendo el extracto metanólico de $B$. laxiflora el que demostró actividad antiproliferaiva del tipo selectivo en las LC cancerosas, debido a que la IC 50 para L929 no pudo ser calculada por no presentar cambios aparentes en la proliferación celular a la máxima concentración evaluada de $200 \mu \mathrm{g} / \mathrm{mL}$ (tabla 3 y 4). No obstante, la resina de $B$. microphylla es la que presenta la mejor actividad antiproliferativa en las cuatro LC cancerosas, en comparación con las otras dos especies del género Bursera, siendo la LC humana de cáncer cervicouterino, HeLa, la más afectada por esta resina, donde solo es necesario $13.8 \mu \mathrm{g} / \mathrm{mL}$ para inhibir el $50 \%$ de la proliferación celular. Otros estudios han llegado más lejos; un claro ejemplo es el aislamiento de compuestos con actividad antiproliferativa de Bursera fagaroides. En $B$. fagaroides se aislaron lignanos que presentan fuerte actividad antiproliferativa contra diferentes líneas celulares. Los lignanos son compuestos fenólicos que presentan actividad antioxidante, que también han sido aislados de B. simuraba, y que pudieran explicar la actividad antioxidante y antiproliferativa de B. laxiflora (Rojas-Sepúlveda et al., 2012).

La resina de $B$. microphylla, y el extracto metanólico de J. macrocarpa ssp. pungens son las únicas dos muestras que presenten $\mathrm{IC}_{50}$ consideradas citotóxicas, con valores menores a $30 \mu \mathrm{g} / \mathrm{mL}$, según lo establecido instituto nacional del cáncer de los Estados Unidos (American National Cancer Institute) (Ogbole, Segun y Adeniji, 2017). 


\section{CONCLUSIÓN}

De las seis especies evaluadas, J. macrocarpa ssp. pungens, tanto del extracto de las partes aéreas como de las cáscaras de fruto, y B. microphylla, poseen la mayor actividad antiproliferativa, y debido a la actividad antioxidante que presentan, es poco probable que los compuestos responsables por la actividad antiproliferativa sean compuestos fenólicos. Por otra parte, B. laxiflora mostró los resultados más interesantes de toda la investigación; la mejor actividad antioxidante de las muestras evaluadas y actividad antiproliferativa del tipo selectivo. Estos resultados, en conjunto con los estudios fitoquímicos reportados para el género Bursera, indican que es probable que los compuestos responsables por la actividad antioxidante y antiproliferativa, en B. laxiflora, sean del tipo lignano. En conjunto, independiente de la actividad antioxidante, las tres especies herbales son una fuente potencial de moléculas con actividad antiproliferativa para LC cancerosas.

\section{REFERENCIAS}

Abbas, M., Saeed, F., Anjum, F.M., Afzaal, M., Tufail, T., Bashir, M.S., Ishtiaq, A., Hussain, S., Suleria, H.A.R., 2017. Natural polyphenols: An overview. Int. J. Food Prop. https://doi.org /10.1080/10942912.2016.1220393

Afsar, T., Razak, S., Shabbir, M., Khan, M.R., 2018. Antioxidant activity of polyphenolic compounds isolated from ethylacetate fraction of Acacia hydaspica R. Parker. Chem. Cent. J. https://doi.org/10.1186/s13065-018-0373-x

Agrawal, S., Kulkarni, G.T., Sharma, V.N., 2010. ORIGINAL ARTICLE A Comparative Study on the Antioxidant Activity of Methanol Extracts of Acacia. Alcohol 4, 78-84.

Aldaco-Sarvide, F., Pérez-Pérez, P., Cervantes-Sánchez, G., Torrecillas-Torres, L., Erazo-Valle-Solís, A.A., CabreraGaleana, P., Motola-Kuba, D., Anaya, P., Rivera-Rivera, S., Cárdenas-Cárdenas, E., 2019. Mortalidad por Cáncer en México: actualización 2015. Gac. Mex. Oncol. https://doi. org/10.24875/j.gamo.m18000105

Bah, M., Gutiérrez-Avella, D.M., Mendoza, S., Castañeda-Moreno, R., Rodríguez-López, V., 2014. Chemical constituents and antioxidant activity of extracts obtained from branch bark of Bursera simaruba. Bol. Latinoam. y del Caribe Plantas Med. y Aromat.

Bray, F., Ferlay, J., Soerjomataram, I., Siegel, R.L., Torre, L.A., Jemal, A., 2018. Global cancer statistics 2018: GLOBOCAN estimates of incidence and mortality worldwide for 36 cancers in 185 countries. CA. Cancer J. Clin. https://doi.org/10.3322/ caac. 21492

Fon-Fay, F.M., Pino, J.A., Hernández, I., Rodeiro, I., Fernández, M.D., 2019. Chemical composition and antioxidant activity of Bursera graveolens (Kunth) Triana et Planch essential oil from Manabí, Ecuador. J. Essent. Oil Res. https://doi.org/10.1 080/10412905.2018.1564381

García-Sosa, K., Sánchez-Medina, A, Álvarez, S.L., Zacchino, S., Veitch, N.C., Simá-Polanco, P., Peña-Rodriguez, L.M., 2011. Antifungal activity of sakurasosaponin from the root extract of Jacquinia flammea. Nat. Prod. Res. 25, 1185-1189. https:// doi.org/10.1080/14786419.2010.511215

Gigliarelli, G., Zadra, C., Cossignani, L., Robles Zepeda, R.E., Rascón-Valenzuela, L.A., Velázquez-Contreras, C.A., Marcotullio, M.C., 2018. Two new lignans from the resin of Bursera microphylla A. gray and their cytotoxic activity. Nat. Prod. Res. https://doi.org/10.1080/14786419.2017.1375922
Karadag, A., Ozcelik, B., Saner, S., 2009. Review of methods to determine antioxidant capacities. Food Anal. Methods. https://doi.org/10.1007/s12161-008-9067-7

Moloney, J.N., Cotter, T.G., 2018. ROS signalling in the biology of cancer. Semin. Cell Dev. Biol. https://doi.org/10.1016/j. semcdb.2017.05.023

Moo-Puc, R., Chale-Dzul, J., Caamal-Fuentes, E., 2013. Bonellia albiflora: A Mayan Medicinal Plant That Induces Apoptosis in Cancer Cells. Evid. Based. Complement. Alternat. Med. 2013, 823453. https://doi.org/10.1155/2013/823453

Ogbole, O.O., Segun, P.A., Adeniji, A.J., 2017. In vitro cytotoxic activity of medicinal plants from Nigeria ethnomedicine on Rhabdomyosarcoma cancer cell line and HPLC analysis of active extracts. BMC Complement. Altern. Med. https://doi. org/10.1186/s12906-017-2005-8

Rojas-Sepúlveda, A.M., Mendieta-Serrano, M., Mojica, M.Y.A., Salas-Vidal, E., Marquina, S., Villarreal, M.L., Puebla, A.M., Delgado, J.I., Alvarez, L., 2012. Cytotoxic podophyllotoxin type-lignans from the stem bark of Bursera fagaroides var. fagaroides. Molecules. https://doi.org/10.3390/ molecules17089506

Sadiq, M.B., Tharaphan, P., Chotivanich, K., Tarning, J., Anal, A.K., 2017. In vitro antioxidant and antimalarial activities of leaves, pods and bark extracts of Acacia nilotica (L.) Del. BMC Complement. Altern. Med. https://doi.org/10.1186/s12906017-1878-x

Sofi, M.S., Nabi, S., Mohammed, C., Sofi, S., 2018. The role of phytocompounds in cancer treatment: A current review. $83 \sim$ J. Med. Plants Stud.

Sridhar, K., Charles, A.L., 2019. In vitro antioxidant activity of Kyoho grape extracts in DPPH [rad] and ABTS [rad] assays: Estimation methods for EC 50 using advanced statistical programs. Food Chem. https://doi.org/10.1016/j. foodchem.2018.09.040

Tirzitis, G., Bartosz, G., 2010. Determination of antiradical and antioxidant activity: Basic principles and new insights. Acta Biochim. Pol. https://doi.org/10.18388/abp.2010_2386

Torres-Moreno, H., 2017. Mecanismos moleculares de triterpenos de Ibervillea sonorae asociados a la muerte celular por apoptosis in vitro e in vivo. Universidad de Sonora.

Torres-Moreno, H., Velázquez, C.A., Garibay-Escobar, A., Curini, M., Marcotullio, M.C., Robles-Zepeda, R.E., 2015. Antiproliferative and apoptosis induction of cucurbitacintype triterpenes from Ibervillea sonorae. Ind. Crops Prod. 77, 895-900. https://doi.org/10.1016/j.indcrop.2015.09.055

Valenzuela-Cota, D.F., Buitimea-Cantúa, G. V., PlascenciaJatomea, M., Cinco-Moroyoqui, F.J., Martínez-Higuera, A.A., Rosas-Burgos, E.C., 2019. Inhibition of the antioxidant activity of catalase and superoxide dismutase from Fusarium verticillioides exposed to a Jacquinia macrocarpa antifungal fraction. J. Environ. Sci. Heal. - Part B Pestic. Food Contam. Agric. Wastes. https://doi.org/10.1080/03601234.2019.1622 978

Vila-Luna, S.E., Moo-Puc, R.E., Torres-Tapia, L.W., PerazaSánchez, S.R., 2017. New metabolites with cytotoxic and antiproliferative activities isolated from Bonellia macrocarpa. Phytochem. Lett. https://doi.org/10.1016/j. phytol.2016.12.016

Yetman, D., Van Devender, T.R., 2002. Mayo Ethnobotany, 1st ed. University of California Press. 